\title{
CHARACTER TABLES DETERMINE ABELIAN SYLOW 2-SUBGROUPS
}

\author{
ALAN R. CAMINA AND MARCEL HERZOG
}

ABstract. A finite group has an abelian $S_{2}$ if and only if every 2-element is 2-central.

In his survey talk at the AMS Summer Institute on Finite Group Theory, Santa Cruz, Califormia, 1979, Walter Feit mentioned the following problem:

Can one read from the character table of a finite group if its Sylow $p$-subgroups are abelian?

The aim of this note is to show that a Sylow $p$-subgroup of $G$ is abelian iff each $p$-element of $G$ is $p$-central, provided that either $p=2$ (Theorem 6 ) or $G$ is $p$-solvable (Proposition 1). Thus in these two cases the answer to Feit's question is in the affirmative. The authors are not aware of finite groups satisfying one of the above-mentioned properties, but not the other. In this note we also show that the property: "a Sylow 2-subgroup of $G$ is elementary abelian" can be read from the character table of $G$ (Corollary 5).

In this note $G$ denotes a finite group. Let $p$ be a prime. The symbol $S_{p}$ stands for "Sylow $p$-subgroup". An arbitrary Sylow $p$-subgroup of $G$ will also be denoted by $S_{p}$. An element $x$ of $G$ is called $p$-central if its centralizer contains an $S_{p}$ of $G$, and it is called real if its column in the character table of $G$ is real. It is well known that $x$ is real iff $x$ is conjugate to $x^{-1}$ in $G$. Finally, let $C_{G}^{*}(x)=\left\{g \in G \mid x^{g}=x\right.$ or $\left.x^{-1}\right\}$. This is a subgroup of $G$ and $C_{G}^{*}(x)=C_{G}(x)$ unless $x$ is a real element satisfying $x^{2} \neq 1$, in which case $\left|C_{G}^{*}(x): C_{G}(x)\right|=2$.

If $G$ is $p$-solvable, we can easily prove the following proposition.

Proposition 1. Let $G$ be a p-solvable finite group. Then $S_{p}$ of $G$ is abelian iff each p-element of $G$ is p-central.

Proof. The "only if" part is trivial. So suppose that each $p$-element of $G$ is $p$-central. By Theorem 3.3 in [3], $G=O_{p^{\prime} p p^{\prime}}(G)$. Now $\bar{G}=G / O_{p^{\prime}}(G)$ is $p$-closed and still satisfies our assumption. Thus $S_{p}$ of $\bar{G}$ is abelian and hence $S_{p}$ of $G$ is abelian.

From now on, we shall deal with the prime $p=2$ and $G$ will denote a group of even order. The following remark is trivial, but basic.

Proposition 2. A nontrivial 2-central element of $G$ is real iff it is an involution.

Received by the editors October 25, 1979.

AMS (MOS) subject classifications (1970). Primary 20C15, $20 \mathrm{D} 99$. 
Proof. Every involution is real. Conversely, let $u \neq 1$ be a 2-central element of $G$. Then $C_{G}^{*}(u)=C_{G}(u)$. Thus, if $u$ is real, it follows that $u$ is an involution.

COROLlaRY 3. The 2-central involutions of $G$ are determined by the character table of $G$.

Corollary 4. An $S_{2}$ of $G$ is elementary abelian iff every 2-element of $G$ is 2-central and real.

COROllary 5. The property "an $S_{2}$ of $G$ is elementary abelian" is determined by the character table of $G$.

Finally, we prove

THEOREM 6. Let $G$ be a finite group. Then $S_{2}$ of $G$ is abelian if and only if each 2-element of $G$ is 2-central.

Proof. The "only if" part is trivial. By Proposition 1 we may assume that $G$ is a nonsolvable group of minimal order such that each 2-element of $G$ is 2-central, but $S_{2}$ of $G$ is nonabelian. Clearly $O(G)=1$ and $O^{2^{\prime}}(G)=G$. If $u$ and $v$ are distinct involutions in $S_{2}$, then $u v$ is a real 2-element, hence an involution by Proposition 2. Thus $\Omega_{1}\left(S_{2}\right)$ is abelian. Moreover, it follows from our assumptions that $F(G)=$ $\mathrm{O}_{2}(G)$ is centralized by each 2-element of $G$, hence $O_{2}(G)<Z(G)$.

Let $E$ be the central product of all subnormal quasisimple subgroups of $G$ and let $F^{*}=O_{2}(G) E$. It is well known that $C_{G}\left(F^{*}\right)=Z\left(F^{*}\right)$ (see $[1, \S 10]$ ), hence $E \neq 1$. Suppose that $G$ is quasisimple. As $\Omega_{1}\left(S_{2}\right)$ is elementary abelian and every 2-element of $G$ is 2-central, it follows by [2] that $G$ is simple with an abelian $S_{2}$, a contradiction. So each quasisimple subnormal subgroup of $G$ is a proper subgroup of $G$, hence has an abelian $S_{2}$. Consequently, $F^{*}=O_{2}(G)^{*} M_{1} * \cdots * M_{r}$, a central product, with $M_{i}$ quasisimple having abelian $S_{2}$. Thus $F^{*}$ has an abelian $S_{2}$ and it suffices to show that if $x$ is a 2-element of $G$, then $x \in F^{*}$. Suppose that $x \notin F^{*}$. Then $x$ acts as an automorphism on $F^{*}$, centralizing $O_{2}(G)$ and permuting the $M_{i}$. However, $x$ centralizes an $S_{2}$ of $F^{*}$, hence $x$ normalizes each $M_{i}$. Thus we may assume that $x$ acts as an outer automorphism of even order on some $M_{i}$, and hence also on the simple group $M=M_{1} / Z\left(M_{1}\right)$ [1, Lemma 10.3]. Since $M$ is a simple group with an abelian $S_{2}$ and with an outer automorphism of even order, it follows by [4] that $M=\operatorname{PSL}(2, q)$, with $q=2^{n}>2$ or $q \equiv 3$ or $5(\bmod 8), q>5$. If $q=2^{n}$ and the order of $x$ as an outer automorphism is $2^{k}=r$, then $C_{M}(x)=$ $\operatorname{PSL}\left(2,2^{n / r}\right)$ and $x$ does not centralize an $S_{2}$ of $M$, a contradiction. If $q \equiv 3$ or 5 $(\bmod 8)$ and $q>5$, then $Z\left(M_{1}\right)=1$, and $x$ acts on $M_{1}$ as an element of $P G L(2, q)$, hence it does not centralize an $S_{2}$ of $M_{1}$, a contradiction. The proof of Theorem 6 is complete.

COROllary 7. The property "an $S_{2}$ of $G$ is abelian" is determined by the character table of $G$.

AdDed In Proof. The authors have been informed that ${ }^{2} F_{4}(2)$ has just one class of 3-elements and its $S_{3}$ is nonabelian of order 27 and exponent 3. 


\section{REFERENCES}

1. T. M. Gagen, Topics in finite groups, Cambridge Univ. Press, London, 1976.

2. D. M. Goldschmidt, 2-fusion in finite groups, Ann. of Math. (2) 99 (1974), 70-117.

3. D. Gorenstein, Finite groups, Harper and Row, New York, 1968.

4. J. H. Walter, The characterizaton of finite groups with abelian Sylow 2-subgroups, Ann. of Math. (2) 89 (1969), 405-514.

Department of Mathematics, University of EAst ANGli, Norwich, ENGland

Department of Mathematics, Tel-Aviv University, Tel-Aviv, Israbl 